\title{
La végétation et la détermination du haut niveau des cours d'eau
}

\author{
The connection betwen vegetation \\ and high water levels in rivers
}

\author{
PAR J. ROUSSEAU, \\ PROFESSEUR ASSOCIE A LA SORBONNE, \\ AUTREFOIS DIRECTEUR DU JARDIN BOTANIQUE DE MONTRÉAL
}

\begin{abstract}
Les communautés végétales sont des réactifs des conditions écologiques, des appareils enregistrant les sautes d'humeur du temps. Entre la végétation «aquatique», exigeant la submersion constante, et la «terrestre», ne tolérant pas l'immersion périodique ou prolongée, se place la formation riparienne qui requiest le bain périodique ou, du moins, le tolère. Ces éléments - comprenant des arbres aussi bien que des arbustes et des plantes herbacées et s'étageant sur plusieurs plans - sont des indicateurs du haut niveau des crues périodiques. Une florule riparienne équilibrée résulte de la fluctuation du niveau pendant une vingtaine d'années au moins. Le haut niveau normal ordinaire, déterminé au moyen de la formation végétale riparienne, coüncide avec la moyenne des crues printanières. C'est un nivean atteint normalement une année sur deux. Comme la flore varie avec la latitude, les conditions édaphiques, divers facteurs écologiques et l'histoire géologique, on ne peut recourir à une « recefte» invariable pour déterminer partout le haut niveaul. Les principes de base étant constamment les mêmes, les espèces indicatrices varieront avec les lieux. A titre d'exemple, l'auteur présente un secteur de la vallée du SaintLaurent, où la converture végétale d'origine n'a pas encore fui devant l'attaque de l'Homme.
\end{abstract}

La plupart des dictionnaires présentent la forêt comme une étendue de terrain couverte d'arbres. Celui qui a vécu sous sa voûte y voit une symbiose incessante: de grands fûts qui portent leur frondaison dans le ciel, des sousbois étagés, une faune en quête de gîte et de pâture, des nuées de parasites, des bactéries qui triturent le sol et aussi l'homme qui en dépend. De même, la définition ordinaire des cours d'eau, «nom donné à toutes les eaux courant à découvert», mériterait d'être révisée ou nuancée. Le géographe, l'hydrologue et le biologiste

\begin{abstract}
Plant life can be looked upon as an "ecological barometer". Aquatic and terrestrial vegetation (the former. always in or under water, and the latter unable to stand even temporary submersion) are extremes; between them, there are various types of riparian vegetation, including a wide range of tree, bush and herbaceous plant species, which either need periodic submersion or, at least, do not suffer from it, and act as "indicators" of periodic flood high water levels. A balanced riparian florula results from water level fuctuations during a period of at least twenty years. The normal high water level inferred from riparian vegetation coincides with the mean spring spate level, and usually occurs every other year. As plant life baries with latitude, edaphic conditions, exologic factors, and geological features, no hard-and-fast rule is available for high water level determinations. Though the basic principles are invariably the same, the "indicator species" vary from one place to the next. The Author quotes the example of a certain part of the Saint Lawrence valley, where the original negetation cover is still unaffected by human activities.
\end{abstract}

y voient une pièce d'eau en mouvement (1), évoluant entre des berges et servant d'habitat à une flore et une faune.

A l'hydrologue et à l'hydraulicien, la végétation pose des problèmes impérieux : la fixation

(1) Malgré les dictionnaires, il est bien difficile de ne pas placer les lacs parmi les cours d'eau, au même titro que les rivières. La plupart font partie des systèmes fluviaux. Ecologiquement, il n'y a guère de distinction nette entre les deux éléments, saut dans le cas des exceptionnels lacs de cratères, qui sont néanmoins soumis à un mouvement vertical de la nappe d'eau. 
du sol des rives, l'obstruction des canaux, souvent par des plantes introduites d'un autre continent, parfois la croissance épidémique d'algues filamenteuses ou de diatomées qui bouchent les filtres des aqueducs, et combien d'autres!

La flore, toutefois, n'est pas toujours pour les ingénieurs une source d'ennuis. Quand le cours d'eau, navigable ou flottable, relève du domaine public, les propriétés riveraines - à défaut de législation contraire - se rendent jusqu'au lit du cours d'eau, limité par le haut niveau normal. Advienne une élévation artificielle de la nappe d'eau, par suite de la construction d'un barrage, les responsables doivent acquérir des riverains tout le terrain submergé au-dessus de l'ancien haut niveau normal. La compilation des statistiques recueillies aux échelles d'étiage pendant une vingtaine d'années permet de le fixer avec précision; mais lorsque les données sont insuffisantes ou que les tribunaux considèrent comme preuves de ouï-dire des cotes établies au cours de décennies passées par des opérateurs qui ne sont plus là pour témoigner, il reste une autre solution : déterminer le haut niveau normal en recourant à l'étagement écologique.

Dans la province de Québec, où grèves et berges conservent habituellement leur couverture végétale spontanée, le procédé a frécquemment servi depuis une trentaine d'années. Son initiateur fut mon maitre, le professeur Marie-Victorin, alors directeur de l'Institut botanique de l'Université de Montréal. La mise au point postérieure de la technique a permis une grande précision, comme on le verra dans la présente étude, qui découle de l'expérience personnelle de l'auteur.

Le climat et le sol différencient les flores. Chaque territoire a ses especes indicatrices. De la zone arctique à l'équateur et d'un continent à l'autre, l'association végétale modifie sa composition. Bien plus, les secteurs d'une même zone présentent des mosaïques végétales différentes : les éléments utilisables à Montréal font défaut à Lyon ou Bordeaux, sous la même latitude, mais à des paliers non équivalents de la zone tempérée. Aussi, en premier lieu, importe-t-il d'aborder des principes généraux. Les éléments précis, présentés systématiquement à la suite, et tous tirés de l'expérience de l'auteur, proviennent de l'est du Canada. La végétation des grèves et des berges, que l'homme y a moins bouleversée qu'en Europe, permettra de dégager des conclusions plus nettes.

\section{LE RYTHME ANNUEL DES COURS D'EAU}

Le cours d'eau de la zone tempérée n'a pas l'humeur capricieuse de la rivière tropicale. Parfois, sous un ciel lourd - une haleine de haut fourneau - un point noir, menacant, se forme à l'horizon. Presque aussitôt, le vent claque comme un fouet, suivi d'un orage torrentiel. Vingt minutes plus tard, partout des ruisseaux, surgis on ne sait d'où, coupent les voies de communication. Au lieu de l'arroyo desséché, une rivière gonflée charriant des sédiments.

Dans les pays tempérés, l'eau des cours d'eau baisse lentement du printemps à l'automne, découvre une grève étroite, soumise à d'imperceptibles pulsations, se fige pour l'hiver, grossit de nouveau à la saison suivante et répète sans défaillir le même cycle. Somme toute, une marée, indépendante de la lune, de l'équinoxe et du solstice, mais qui, biologiquement, se comporte comme celle des estuaires d'eau douce. Les deux habitats, d'ailleurs, hébergent des hôtes communs.

La variation du débit des cours d'eau relève de facteurs climatiques. Dans les pays à neiges abondantes, l'eau des hivers, fossilisée en cris- taux, mollit au printemps sous l'attaque plus directe des rayons solaires. En quelques jours, le sol se dégage de sa gangue hivernale. Le tapis moussu des forêts fait bien ce qu'il peut pour retenir le liquide; le sol encore figé en absorbe une faible part, mais le reste coule en surface: les petits filets font les ruisseaux; les ruisseaux, les grandes rivières, les rivières, les fleuves et les mers. Comme le bombardement solaire sur le sol gelé se produit partout à la fois, la rivière grossit dans la vallée sans sortir de son auge; à moins que des glaces flottées ne causent des embâcles ou que des pluies d'exception ne viennent s'ajouter aux neiges fondues.

Le rythme saisonnier des lacs et des rivières, depuis les basses eaux de l'été jusqu'aux crues printanières, est une fluctuation aussi normale que la course des aiguilles sur le cadran de l'horloge. Chez l'homme, la pulsation du cour, d'un âge à l'autre, oscille entre des normes; de même, le pouls de la rivière fluctue entre d'étroites limites au cours d'années consécutives. Comme le rythme des saisons, celui des ans est un phénomène naturel. Dans une période météo- 
rologique normale - une vingtaine d'années de suite - la rivière atteint habituellement la mesure de sa taille. Le plus souvent toutefois, elle gagne une cote moins élevée - le haut niveau normal moyen - que l'on peut retenir en pratique pour délimiter le lit du cours d'eau; mais, en réalité, celui-ci se rend jusqu'au haut niveau normal absolu - le plus haut point atteint par l'eau, libre de tout embâcle, au cours d'un cycle météorologique.

La compilation des maximums atteints annuellement, pendant une vingtaine d'années consécutives, suffit d'habitude pour fixer avec précision le haut niveau normal moyen; mais dans les endroits où les données hydrologiques font défaut, la végétation peut fournir une réponse. La flore se comporte comme l'appareil enregistreur des sautes d'humeur du temps. Chaque espèce a ses exigences ou ses caprices. La forêt dense ne saurait que faire des plantes de pleine lumière. Certaines affectionnent les longs jours de soleil estivaux de la toundra arctique, et pour cela font défaut sur les sommets alpins des tropiques. Des végétaux sont frileux, d'antres poussent la tête à travers des couches de neige. L'affinité pour l'eau est non moins spécifique. Entre les plantes aquatiques, subissant un bain continuel, et celles des déserts, humectées seulement de rosée matinale, s'en situe une gamme au comportement varié. La flore terrestre accepte volontiers les ondées et sa composition dépend beaucoup de la chute annuelle de pluie; mais la submersion lui est habituellement fatale en dehors du sommeil hivernal. Les terrains submergés régulièrement ont une flore autochtone. Le bain annuel repousse quelques espèces, et favorise un tapis végétal sur mesure qui raconte l'histoire des fluctuations séculaires.

Vous promenant en canot, au cours de l'été, à distance de la rive, vous distinguez dans le ciel la vague ondulante du faite des arbres, quand la base de la végétation longe parallelement la surface de l'eau. Bien plus, la grève présente des strates horizontales nettement délimitées. Les florules ripariennes sont des archives qui enregistrent la chronique des variations du niveau de l'eau. Ces documents, accumulés par le travail des siècles, permettent de retrouver, à peu de centimètres près, l'amplitude nor-

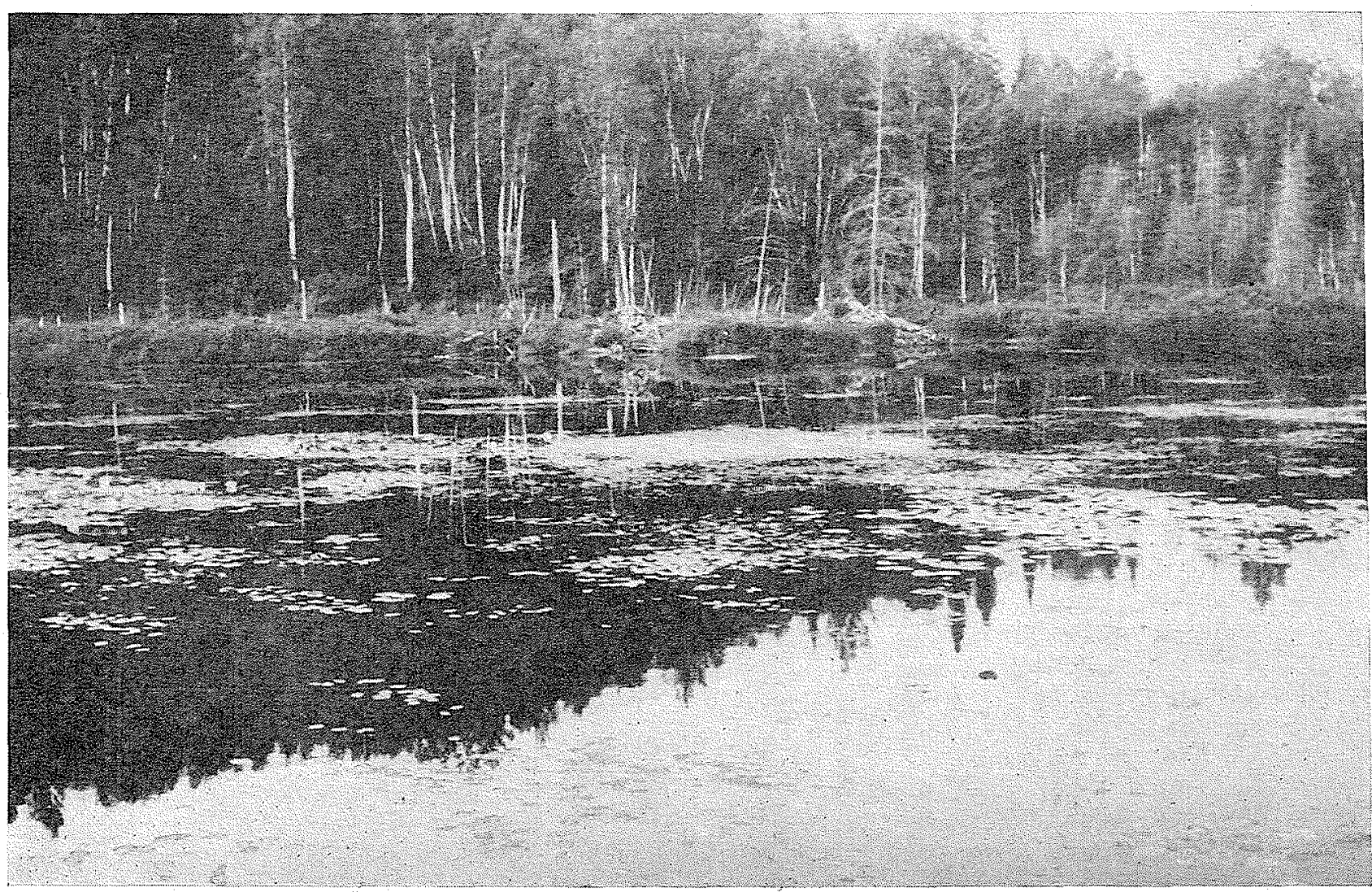

FIG. 1

La rive d'un cours d'eau, vue à distance, présente souvent des strates de végétation nettement horizontales : elles sont la résultante des changements périodiques de nivean. 
male du cours d'eau. Les cotes de niveau basées sur la végétation concordent remarquablement avec celles des échelles d'étiage.

Les débordements causés par des embâcles de frazil - ces aiguilles de glace si caractéristiques des cours d'eau rapides des hivers très froids ont une action très faible sur la composition du tapis végétal qui somnole alors. Les inondations printanières, provoquées par les barrages de glaces floltées - à une époque où le sol gelé n'a pas encore permis aux bourgeons d'éclater ont le même comportement. Les tissus délicats sont alors protégés par la carapace imperméable des écailles. L'activité des plantes vivaces, encore extrêmement réduite, se produit en vase clos. On sait d'ailleurs que les bulbes, les tubercules, conservés dans des caves fraîches, ne souffrent pas à cette époque. Bien plus, à la même sai son, des arbustes peuvent survivre un certain temps sous des abris, hors du sol, leurs racines simplement enveloppées pour les protéger contre la dessiccation. Si l'eau envahit paisiblement l'habitat terrestre pendant Ie repos hivernal, les plantes se comportent alors comme des scaphandriers dans l'élément liquide. En pratique, l'inondation transporte des débris, lacère les plantes et provoque l'érosion, même à l'intérieur des taillis, mais ne crée pas des formations de grèves.

Sur le littoral, il importe de bien distinguer entre la berge et la grève. La sculpture des vallées, variant avec la topographie, résulte d'un long passé géologique et doit souvent sa physionomie aux glaciers - des rivières de glace qui les ont rabotées au début du quaternaire. Le lit de la rivière couvre rarement tout le fond de la vallée. De même, les formations Iittorales se répartissent en deux zones foncièrement distinctes : la grève, partie du lit de la rivière, qui subit le bain annuel imposé par la fluctuation normale, et la berge, plus ou moins escarpée, située au-dessus de la ligne de haut niveau et en dehors du lit.

Tous les habitants de la grève ne sont pas semi-aquatiques. Le milieu possède aussi ses picque-assiettes, venus s'attabler sans invitation. Des mauvaises herbes cosmopolites - pour la plupart des plantes annuelles de croissance rapide - se terrent jusqu'au retrait de l'eau importune pour commencer alors leur vie éphémère. Sur le gravier des rivières d'Anticosti, exondé pendant l'été, poussent des aristocrates de la montagne et de la toundra arctique, sans affinité pour l'eau, mais attirés par la pleine lumiere que refuse la forêt voisine, et tolérant cet habitat grâce à leur cycle estival court et à leurs accumulations de réserves souterraines.

Ces intrus ou invités, étrangers au milieu, ne décident pas de l'économie de la maison. Les autres, par contre, permettent de distinguer quatre niveaux différents : le plus haut, que l'on perçoit à l'occasion, résulte de l'action des vagues de tempêtes et des rares inondations survenues au début de la période de croissance; le second, plus has - le haut niveau normal absolu - borne véritablement le lit du cours d'eau. Vient ensuite le haut niveau normal moyen, beaucoup plus facile à déceler au moyen de la couverture végétale. En dehors des domaines comportant des droits de grève ou la propriété en eau profonde (utilisation des battures el des plages, exploitation de la pêche littorale, droits de flottage, construction de quais, jetées ou barrages, etc.), la ligne de haut niveau normal moyen - en pratique, mais non en droit - limite très souvent la propriété des riverains, d'un côté, et, de l'autre, le lit de la rivière relevant du domaine public. Enfin, le bas niveau normal, également décelable au moyen de la végétation - quoique moins clairement souvent que le haut niveau normal. Il faut en tenir compte

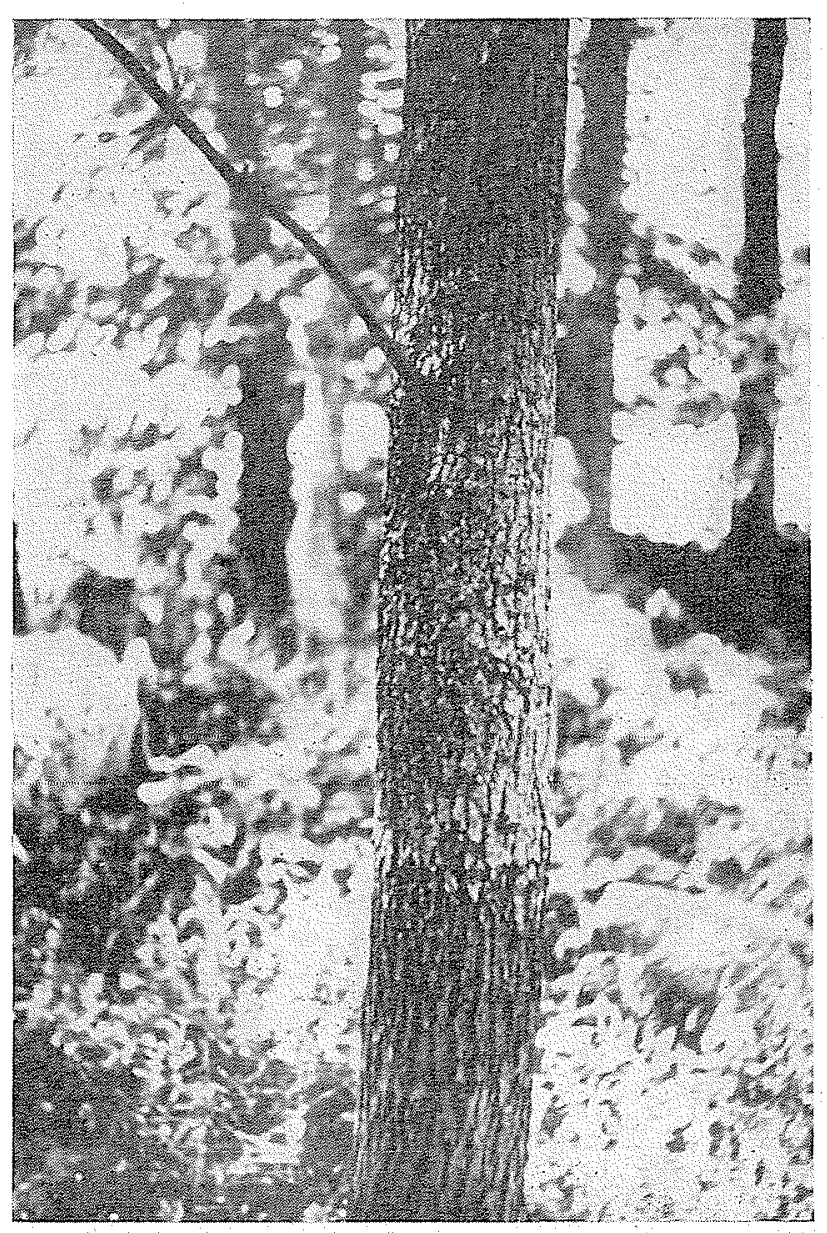

Fic. 2

Manchon de lichens (partie grisatre dans la partie centrale du tronc). La croissance s'est arrêtée brusquement au contact de l'eau. 

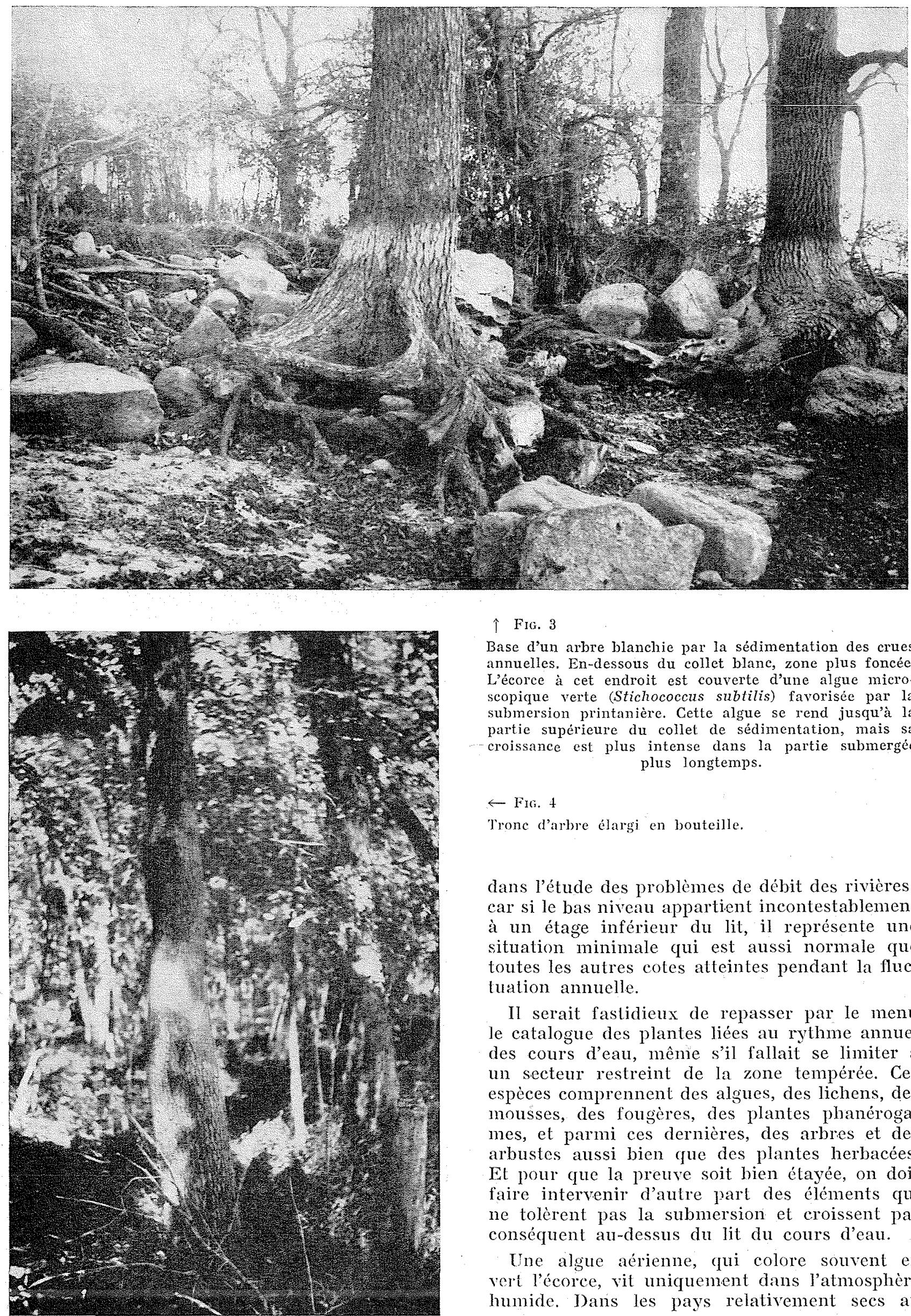

$\uparrow$ FIG. 3

Base d'un arbre blanchie par la sédimentation des crues annuelles. En-dessous du collet blanc, zone plus foncée. L'écorce à cet endroit est couverte d'une algue microscopique verte (Stichococcus subtilis) favorisée par la submersion printanière. Cette algue se rend jusqu'à la partie supérieure du collet de sédimentation, mais sa croissance est plus intense dans la partie submergée plus longtemps.

$\leftarrow$ Fyc.

Trone d'arbre élargi en bouteille.

dans l'étude des problèmes de débit des rivières; car si le bas niveau appartient incontestablement à un étage inférieur du lit, il représente une situation minimale qui est aussi normale que toutes les autres cotes atteintes pendant la fluctuation annuelle.

II serait fastidieux de repasser par le menu le catalogue des plantes liées au rythme annuel des cours d'eau, mêne s'il fallait se limiter à un secteur restreint de la zone tempérée. Ces espèces comprennent des algues, des lichens, des mousses, des fougères, des plantes phanérogames, et parmi ces dernières, des arbres et des arbustes aussi bien que des plantes herbacées. Et pour que la preuve soit bien étayée, on doit faire intervenir d'autre part des éléments qui ne tolèrent pas la submersion et croissent par conséquent au-dessus du lit du cours d'eau.

Une algue aérienne, qui colore souvent en vert l'écorce, vit uniquement dans l'atmosphère humide. Dans les pays relativement secs au 


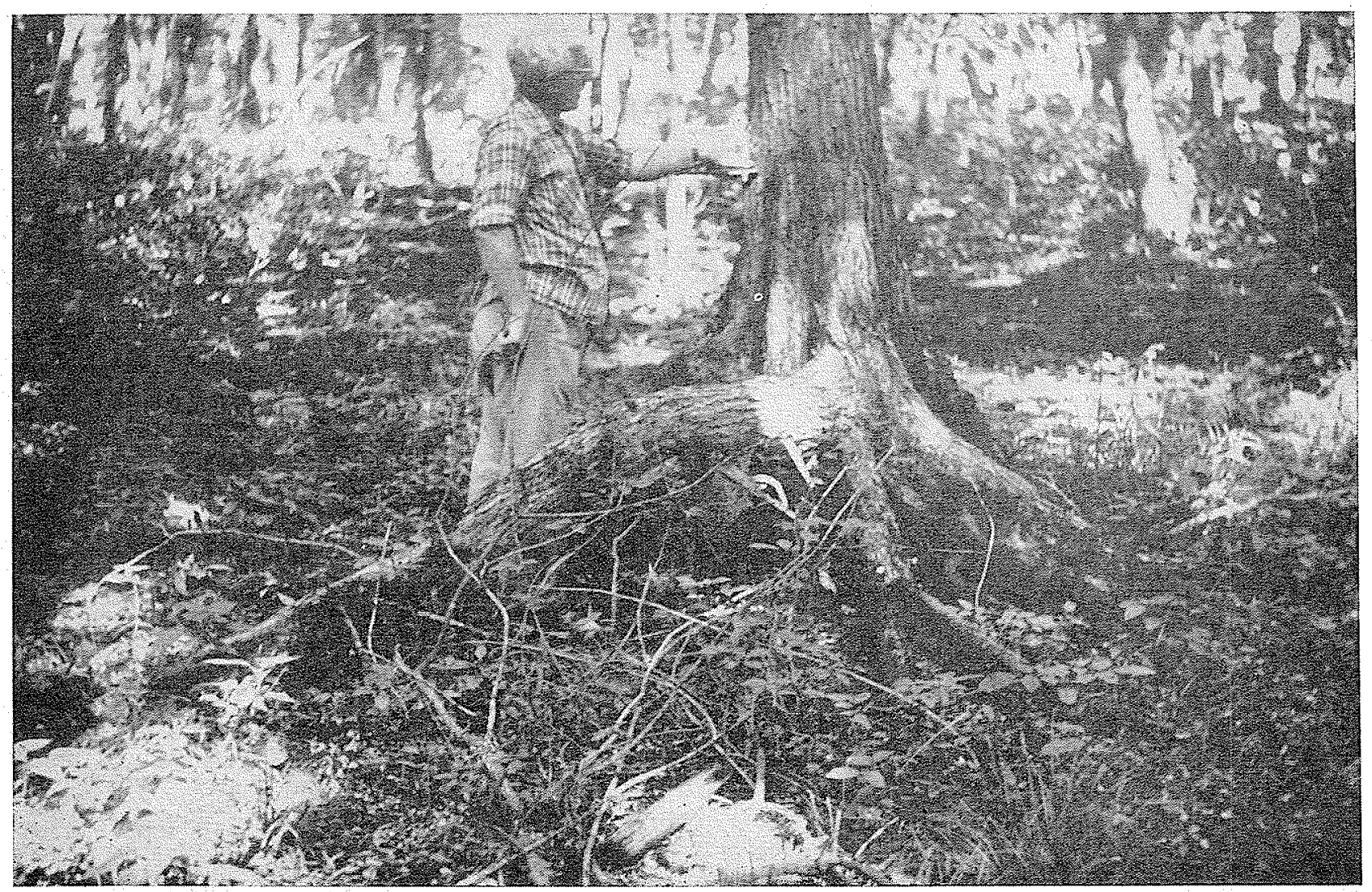

FIG. 5

Orme sur échasses, dans un secteur de forèt submergé au printemps. Les racines sont de véritables ares-boutants qui supportent l'arbre.

cours de l'été, comme la vallée du Saint-Laurent, l'habitat idéal semble une strate étroite dominant d'un mètre ou deux le haut niveau normal. Par contre, dans l'atmosphère plus humide de certains secteurs de la France, l'arbre entier se colore en vert, même à distance des pièces d'eau. Comme de nombreux lichens, ces algues se contentent de boire l'humidité du ciel et les gouttes de pluie. Sur le tronc des arbres bordant les rivières québécoises, ces diverses plantes ne poussent jamais en bas du point d'atteinte de l'eau. Lorsque du large on embrasse du regard la rive, se distingue une ligne continue qui marque le point inférieur de leur croissance. D'autres algues et lichens corticaux, par contre, acceptent une courte submersion. Des mousses construisent un manchon à la base des arbres, dans la zone verticale submergée annuellement, quand d'autres espèces apparentées n'ont pas les mêmes exigences.

Des arbustes, des plantes herbacées sont des commensaux exclusifs de la grève, se distribuant le plus souvent suivant des strates particulières soumises à une submersion de durée définie. La forêt n'est pas un habitat exclusivement terres- tre, et elle empiète parfois sur la grève. Des marécages méridionaux, qui n'assèchent jamais, renferment des arbres qui leur sont propres et dont le trone est fréquemment élargi en bouteille. Des arbres de la vallée du Saint-Laurent - comme les frênes - se comportent souvent de même. Des arbres et des arbustes palustres des pays tropicaux semblent parfois grimpés sur des échasses, tellement leur système radiculaire dépasse l'eau on la boue. J'en ai vus présentant la même physionomie dans les forêts bordant l'Outaouais (est du Canada). Le nord des Etats-Unis recèle des forêts d'érables rouges dans des marais permanents. Le Québec compte au moins deux écotypes de l'espèce, celui des érablières assez bien drainées, et celui des marécages de la région de Montréal. Dans le même secteur du Saint-Laurent, l'érable argenté et le liard du Canada poussent spontanément sous la ligne des hautes eaux printanières. Les graines d'érable argenté germent normalement en bordure de l'eau. Au cours de l'été, la ligne presque ininterrompue des plantules rougeâtres en indique le niveau à l'époque où l'arbre a libéré ses samares à vol hélicoîdal. L'étendue des forma- 
tions spontanées d'une espèce n'indique pas nécessairement la limite de tolérance. On cultive dans les villes tempérées des fleurs de montagnes ou de rivages salés; de mème, les arbres propres aux grèves peuvent pousser avec succès hors de leur milieu naturel quand l'homme favorise leur multiplication.

La spécialisation de l'habitat riparien n'est pas néanmoins un facteur d'uniformité : comme dans la forêt variée, la lutte pour la vie, les conditions édaphiques et les normes climatiques imposent à la grève une physionomie variant avec les lieux. Les eaux qui ont baigné les rives arides des laes laurentiens, où la perchaude au printemps suspend les volutes spiralées de sa fraye parmi les arbustes Jitloraux, rencontreront dans leur course les tables calcaires ou gréseuses des rapides, les schistes usés, les battures de foins coupants, où canards et sarcelles se restaurent pendant la migration, et, après avoir connu le rythme quotidien des estuaires d'eau douce, termineront leur royage parmi les sables halophytiques, couverts de plantes grasses et de seigle de mer.

L'homme lui-mème n'est pas resté étranger au rythme saisonnier des cours d'eau. Pour la chasse, il construit ses affutts au bord de la batture. Lors de la baisse, les bestiaux viennent paître le foin de grève. Sur les îlots bas - - les «communes» dans le Québec - se groupent parfois pour l'été les troupeaux du voisinage. Les grandes prairies de salicaire du lac SaintPierre, sur le Saint-Laurent, fournissent un miel brun d'un arôme et d'une saveur insurpassables. L'herbe-á-lien et le roseau édifiaient la toiture des granges. Et longtemps avant la venue du Blanc en Amérique, l'ojibway cueillait pour sa subsistance la folle avoine des prairies toujours submergées. Depuis un demi-siècle, des secteurs de la grève se couvrent de demeures estivales, dont certaines, juchées sur des échasses, rappellent pendant la crue printanière le palafitte préhistorique.

L'homme n'a pas seulement profité du rythme saisonnier des rivières. La coupe des forêts, qui retenait longtemps la pluie dans ses mailles, en a modifié le régime. Les barrages ont haussé les anciennes rives et reporté plus haut ses pulsa-

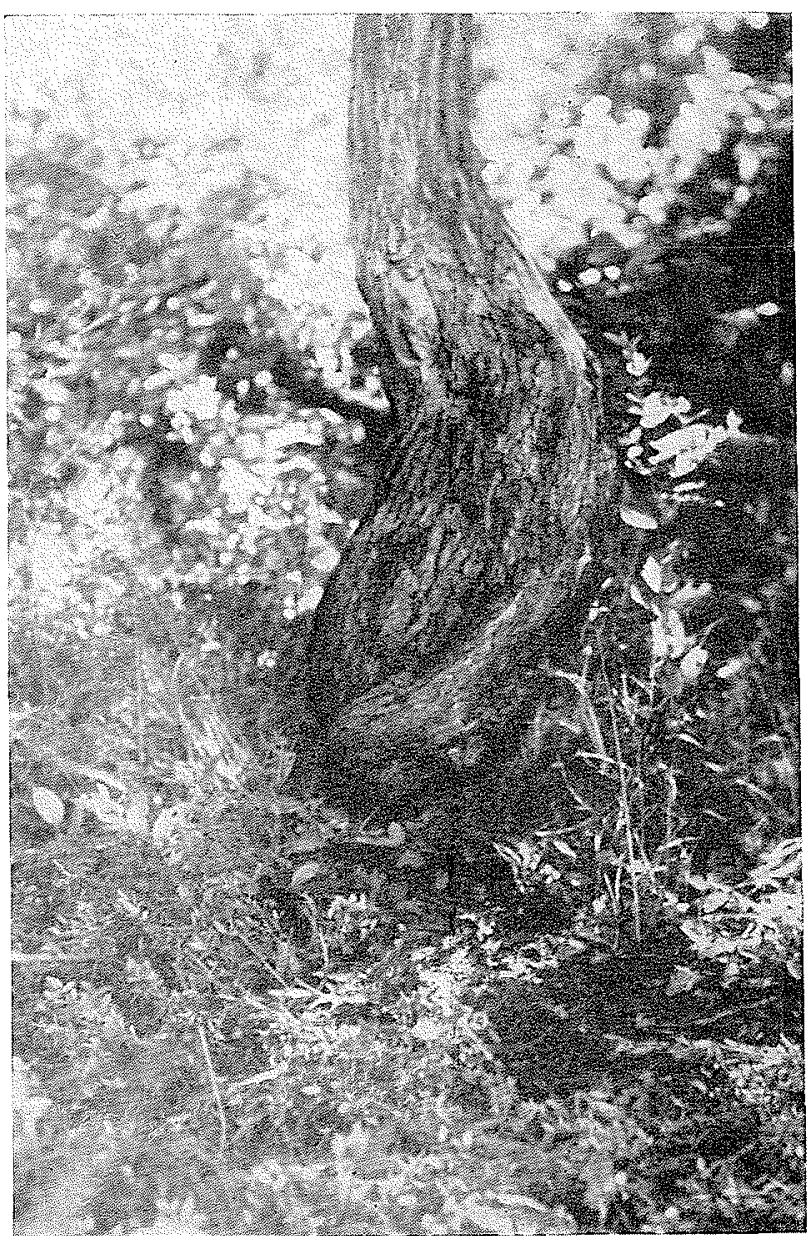

Fig. 6

La base des arbres submergés présente fréquemment ce trone bossué, tordu. Ce faciès est particulièrement dû à l'action des glaces lors des crues.

tions. En petit, l'espèce humaine a créé ce que le temps, à l'échelle géologique, avait fait en grand. D'anciennes rivières sont comblées; la mer Champlain, après la glaciation, abandonne ses terrasses plus de deux cents mètres au-dessus du Saint-Laurent; la Nouvelle-Ecosse abaisse ses tourbières au niveau du flux marin. De nouvelles rivières entrent en scène, mais toujours se continue, comme dans des artères, la pulsation saisonnière qui commande la flore.

\section{LA DÉTERMINATION DU HAUT NIVEAU NORMAL DANS L'EST DU CANADA}

A titre d'exemple, prenons un secteur limité d'une rivière de la région de Montréal. La méthode employée pour y déterminer le haut niveau normal peut s'appliquer aussi bien à d'autres lieux, à condition que les rives soient couvertes d'une végétation spontanée; toutefois, les espèces indicatrices — et même les techniques d'approche - varieront avec les caprices 
de la végétation, soumise aux climats et aux terrains. Aucune "recette invariable» ne peut convenir partout. La population des grèves se comporte comme celle des villes : le costume change avec les lieux et les jours; cependant, les citadins de pays différents, mais soumis à des conditions climaticues semblables (soit neise, soit orage, ou ciel froid ou chaleur accablante), présentent déjà sur une photo une certaine parenté climatique, décelable par la tenue vestimentaire fonctionnelle et partant comparable. Il ne suffit pas de se limiter à quelques éléments pour caractériser le facies végétal d'un habitat; il faut faire appel à l'ensemble de la communauté et tabler même sur des plantes qui font défaut. Chaque secteur de grève peut se comparer à un livre sur un rayon de bibliotheque : quiconque en lirait quelques pages seulement ne pourrait guère se faire une idée précise du contenu de l'ouvrage.

Pour relever le haut niveau normal d'un cours d'eau, l'échelle d'étiage reste le moyen le plus simple à manier, pourvu que l'on puisse compter sur les données d'une vingtaine d'années consécutives. A défaut de ces cotes, ou pour leur apporter une confirmation, la formation végétale de la grève - résultante de la variation saisonnière et annuelle de nombreuses années - apporte une solution précise.

\section{I. - Les niveaux principaux que l'on détermine au moyen de la végétation sont au nombre de quatre}

La communauté végétale permet en outre de déceler des niveaux intermédiaires, indicateurs de débits particuliers el de moments de l'histoire anmuelle de la flore: mais il n'en sera pas queslion à la suite.

1. Niveau d'atteinte des tempêtes. - Point situé au-dessus du lit du cours d'eau, sur le terrain en bordure de la grève, la plus haute

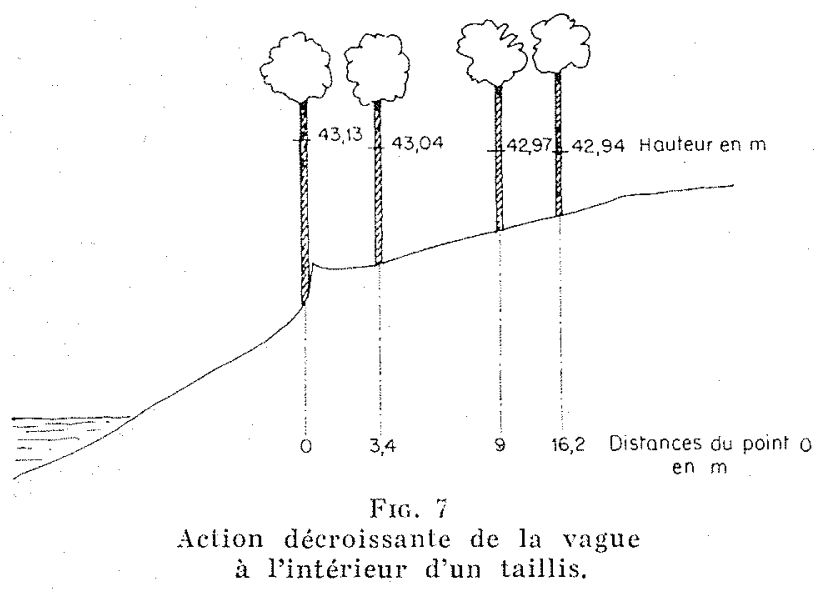

élévation atteinte par les vagues de tempête lorsque le débit de la rivière est au maximum. Là où la pente du fleuve favorise un fort courant, il est illusoire de rechercher ce niveau. Par contre, dans les régions à courant lent, surtout les élargissements lacustres, le vent dominant gonfle la houle qui déferle parfois hors du lit. Dans les cas étudiés par l'auteur, le niveau s'établissait sur des éléments de même ordre que le haut niveau absolu (comme la limite inférieure du manchon de certains lichens corticoles), mais ils étaient beaucoup moins nombreux. L'action érosive de la vague confirmait d'ailleurs le témoignage botanique. A des sites très rapprochés, mais protégés contre le vent, on ne trouvait plus trace de ce niveau. Le cas le plus caractéristique a été celui de forêts basses au bord de la rivière. Les arbres les plus rapprochés de l'eau, immédiatement après la concavité de la grève, avaient subi l'action de l'eau jusqu'à une hauteur de $43,13 \mathrm{~m}$ (au-dessus du niveau de la mer, du réseau géodésique) comme en témoignait un manchon de lichens descendant jusqu'à ce point; à l'intérieur du taillis, par contre, de 3 à $16 \mathrm{~m}$ de la ligne de rivage, là où l'action de la vague brisée se manifestait moins, le manchon de lichens descendait graduellement $(43,04 \mathrm{~m}$ puis $42,97 \mathrm{~m}$ et $42,94 \mathrm{~m}$, voir croquis). En pratique, le niveau d'atteinte des vagues peut se confondre avec le suivant, d'où la nécessité d'établir des stations d'étude dans quelques sites protégés contre le vent. Ceux-ci fourniront des données permettant d'apporter un facteur de correction aux autres endroits.

2. Haut niveau normal absolu. - C'est le plus haut point atteint par l'eau dans un cycle normal, abstraction faite des vagues de tempète et des embâcles. Le haut niveau normal absolu se détermine le mieux là où ne souffle pas de vent dominant vers la rive, et où le courant rapide laisse peu de prise à la vague. Dans la partic supérieure de la grive, se réfugient les espèces tolérant seulement une submersion brève et provenant de graines normalement disséminées par le courant. Elles pourraient pousser dans des habitats «terrestres», mais elles trouvent un terrain d'élection en pleine lumière, au bord de l'eau. Pour la même raison, plusieurs de ces plantes poussent volontiers sur la berge au-dessus du haut niveau normal absolu. La détermination de ce niveau exige l'étude de la formation au complet; elle ne pourrait reposer sur quelques éléments seulement; mais une fois l'étude úlaborée terminée pour quelques sites, il suffit souvent de retenir un petit nombre d'espèces caractéristiques. L'étude doit aussi tenir compte des espèces intolérantes à la submersion et qui croissent uniquement au-dessus du haut niveau normal absolu. On en trouve à tous les degrés 
de la classification. C'est le cas d'une algue aérienne et de lichens poussant sur les écorces. Des Parmelia ne peuvent tolérer une submersion de quelques jours. Le haut niveau normal absolu marque véritablement la limite supérieure du lit du cours d'eau; el Iorsque le lit de la rivière et les terrains voisins appartiennent à des propriétaires différents - souvent le domaine public dans le premier cas --, ce haut niveau absolu devrait constituer normalement la ligne de démarcation. Il n'est pas loisible au riverain de construire des quais, chaussées et demeures plus bas, à moins d'entente préalable avec l'autorité. La coutume, dans le Québec, veut, par contre, que la grève herbeuse exondée serve au pacage des bestiaux à la fin de l'été. Le proprićtaire riverain pourra même continuer parfois la cloture jusqu'à l'eau pour empêcher le bétail de s'éloigner, sans que cela ne lui confère de droits sur la parcelle exondée. Les anciennes concessions de terrains sous le régime seigneurial comportent parfois pour l'acquéreur la possession exclusive des battures pour fins de fauchage, pacage et pêche (pêche en claies de fascine). Simple usufruit toutefois, qui ne doit pas limiter la libre circulation sur la grève et l'accostage.

3. HaUt niveau Normal ordinarre ou Moyen. - Cette ligne de niveau, le long des cours d'eau da Québec, est toujours caractéristique et relativement facile à déterminer au moyen de la végétation spontanée. On doit la baser sur l'ensemble du tapis végétal et non sur quelques especes seulement, quoique certaines parfois se révelent d'excellents indicateurs. Celles-là se décèlent après une étude élaborée de la formation. Les espèces ripariennes comprennent des éléments palustres, semi-palustres ou semi-aquatiques et indifférents; certains sont même des anmuelles qui ne tolèrent pas la submersion, mais il leur suffit de quelques semaines pour passer de la germination à la fruclification. Chaque fois que l'auteur a déterminé le haut niveau normal ordinaire au moyen de la végétation, une comparaison ultérieure avec les cotes de niveau provenant d'échelles d'étiage révélait une concordance presque parfaite. Le haut niveau normal ordinaire correspond à un point atteint par les crues au moins une année sur deux, pendant une vingtaine d'années consécutives. C'est le temps minimal nécessaire pour l'édification d'une formation spontanée de grève. Lors de l'élévation du niveau d'un cours d'eau par une chaussée, le propriétaire de l'œuvre doit acquérir des riverains le terrain au-dessus du haut niveau normal qui doit être submergé. Le haut niveau normal ordinaire constitue alors une excellente ligne de compromis, nettement à l'avantage du riverain. Toutefois et la végétation le démontre clairement -, ce niveau correspond à un point moyen du lit. Les nombreux éléments permettant de fixer cette ligne, dans le secteur choisi comme exemple, font l'objet de notes plus bas.

4. Bas nIveau Normal. - Atteint dans le Québec à la fin de l'été, en août ct septembre, ce niveau indique un palier aussi normal que le haut niveau. Il a fréquemment une importance pratique, parce que la nappe phréatique, à proximité de la rivière, est fréquemment liée au débit du cours d'eau. En dessous du bas niveau normal, croissent des espèces strictement aquatiques, exigeant une submersion régulière. Certaines, comme les nénufars, tolèrent une émersion de quelque durée, sans se flétrir, pourvu que le terrain reste boueux. Sauf cette restriction, les espèces aquatiques, toujours nettement caractérisées dans les Flores, peuvent toutes servir d'indicateurs du bas niveau. Ce niveau, à ma connaissance, n'a guère été invoqué devant les tribunaux, mais il pourrait l'être notamment dans des problèmes relatifs aux aquedues et aux égouts. Il en serait de même lors de l'assèchement d'un cours d'eau qui affecterait la nappe phréatique. En effet, la baisse, comme l'élévation, de cette nappe, peut modifier parfois les conditions écologiques des terrains voisins, en les améliorant ou en les détériorant.

\section{II. - Les espèces indicatrices du haut niveau normal}

Les plantes indicatrices peuvent être de deux sortes :

a) Des espèces ripariennes proprement dites, qui doivent être submergées ou peuvent l'être plus ou moins longtemps sans inconvénient;

b) Des plantes intolérantes à la submersion et croissant au-dessus du haut niveau normal.

La tolérance ou l'intolérance à la submersion varie d'une espèce à l'autre. Leur distribution sur la grève caractérise même parfois des niveaux différents. Il est illusoire de baser un jugement. sur deux ou trois éléments seulement: il faut une étude exhaustive de la flore spontanée du secteur riparien et des habitants situés au-desstis. Les espèces en cause dans l'exemple présenté ici, sont les suivantes.

1. Algues. - Les espèces fixées, colorant en vert le tronc des arbres, peuvent fournir des indications. Le Phytoconis botryoüdes (ou Protococcus viridis), dans la région de Montréal, est une espèce aérienne poussant d'habitude sur l'écorce constamnent émergée, surtout au bord des cours d'eau, où elle forme un manchon vert d'environ un mètre de haut. Si elle ne dépasse 
pas cette strate, au bord du Saint-Laurent, c'est probablement que l'humidité constitue une nappe beaucoup plus dense à la base que plus haut. Le Phytoconis croît exceptionnellement en bas du haut niveau normal, mais toujours associé alors au Stichococcus subtilis, une autre algue verte, également microscopique. La zone d'élection de cette dernière se trouve en dessous du haut niveau normal, dans la strate émergeant après la période des crues. Les manchons des deux algues se distinguent bien, d'autant plus que la base du trone, couverte de Stichococcus subtilis, est blanchie par les sédiments charriés par le fleuve. Des algologues comme Francis Drouet se sont dernandés si les deux algues précitées ne seraient pas des formes écologiques de la même espèce.

2. Lrchens. - La croissance des lichens est très lente et beaucoup plus que celle des phanérogames herbacées. Des espèces sont particulièrement sensibles à la submersion. Ainsi des Parmelia el Physcia, dont les nombreuses espèces toutefois ont chacune leurs exigences. Les seules susceptibles de nous intéresser croissent sur les troncs d'arbres, les rochers et les murs qui ne bougent pas lors des crues ou de la débâcle des glaces ou des gels hivernaux. Arrive une crue exceptionnelle de quelques jours au printemps : le secteur immergé des troncs d'arbres, sarni antérieurement d'une couche intense de lichens, ne comprendra plus désormais que des spécimens morts, faciles à distinguer au cours de l'été. Les lichens submergés succombent à l'asphyxie. De même, les fumées sulfureuses semblent s'opposer à leur croissance, comme on peut s'en rendre comple sur l'Outaouais, à proximité des pulperies. On sait d'autre part les lichens très sensibles aux fumées des villes. En bordure des cours d'eau - Ies conditions de luminosité ou autres étant égales _-, le niveau inférieur du manchon continu de lichens marque habituellement le niveau supérieur d'atteinte de l'eau dans un cycle météorologique normal.

3. Bryophytes (mousses et hépatiques). - Des hépatiques corticoles, comme le Frullania eboracensis, ont le même comportement que les Parmelia précités. Des mousses, par contre - des Leskea et Dichelyma notamment - enveloppent la base des arbres et sont caractéristiques des terrains submergés au printemps. La submersion temporaire peut être due aux crues ou, dans les légères dépressions des bois, aux étangs fugaces produits par la fonte des neiges. Le Leskea obscura et le Dichelyma pallescens indiquent une submersion plus prolongée que le Leskea polycarpa.

4. Ptéridophytes (fougères, prèles et lycopodes). - Les Lycopodinm du Québec sont tous d'habitats «terrestres», sauf le Lycopodium inundatum des tourbières et des grèves acides. La Selaginella rupestris croit sur les rochers sees, tandis que la Selaginella selaginoides habite les tourbières et les grèves acides de l'est et du nord de la province. Beaucoup de fougères québécoises sont caractéristiques des endroits non submergés, mais Dryopteris Thelypteris var. pubescens, Onoclea sensibilis, Osmunda regalis, notamment, sont des commensaux fréquents des grèves montréalaises submergées lors des crues. Les Isoetes sont tous aquatiques, mais tolèrent une certaine émersion lorsque le terrain reste boueux. Des prèles (Equisetum fluviatile, $E$. littorale et $E$, palastre) habitent les marais permanents ou du moins les endroits submergés longtemps chaque année, et restant marécageux après l'émersion. L'E. variegatum est un commensal ordinaire des grèves et il en existe une variété (var. anceps) qui n'habite que la zone intercotidale des estuaires d'eau douce (notamment la région en aval de Québec). Quant à l'E. arvense, on en connait un grand nombre d'écotypes, dont certains caractéristiques des endroits submergés une partie de l'année.

5. Phanerogames : arbres. - La division des phanérogames en arbres, arbustes et plantes herbacées, au lieu de familles naturelles, facilite ici l'étude du problème, et cela d'autant plus que l'on croit fréquemment que la ligne des arbres marque nécessairement la limite supérieure du lit des rivières. C'est généralement le cas dans le Québec, mais il y croît néanmoins des arbres qui vivent dans l'eau --, comme plus au sud aux Etats-Unis. Les Nyssa et Taxodium de la Georgie et de la Floride baignent normalement dans l'eau des marécages. Les mangroves des rives tropicales vivent dans l'eau salée. Aux Ftals-Unis et dans le Québec, un écotype de l'érable rouge (Acer rubrum) adopte les marais. Dans la région de Montréal, l'érable argenté (Acer saccharinum) et le liard du Canada (Populus deltoüdes) poussent spontanément sous la ligne de haut niveau, mais on peut les planter avec succès hors de cet habitat. L'érable rouge (Acer rubrum) et les frênes (dans la région de Montréal, Fraxinus nigra et $F$. pensylvanica, beaucoup plus hydrophyles que $F$. americana) sont fréquemment des arbres ripariens. Les chènes de la région montréalaise peuvent atteindre spontanément la grève et avoir la souche quelque peu immergée lors des crues. Des espèces de la région (Quercus borealis, Q. bicolor, $Q$. alba et $Q$. macrocarpa), les trois dernières sont les plus tolérantes à l'eau, semble-t-il. La partie moyenne de la grève héberge normalement des pousses de chêne, qui, pour la plupart, sont éliminces dans la lutte pour la vie; seules subsistent une partie des plantules de la partie supérieure de la grève. L'orme blanc 
(Ulmus americana), caractéristique de la plaine alluviale du Saint-Laurent - , possede un système de racines superficiel qui lui permet de vivre aux endroits où la couche de terrain essorée l'été est relativement mince. Cette condition permet a l'arbre de croitre en bordure des rivages et d'avoir le système radiculaire inondé au printemps, surtout avant la reprise de la végétation. Le moindre retrait des eaux laisse les racines superficielles au-dessus de la nappe liquide. L'arbre toutefois ne pourrait s'accommoder d'une submersion continuelle. Le peuplier baumier (Populus balsamifera ou $P$. tacamahacca) et le saule fragile (Salix fragilis), introduit d'Europe, ont fréquemment le même comportement que le liard du Canada et l'érable argenté. Dans la partie moyenne de la grève, surgissent fréquemment des pousses de trembles (Populus tremuloüdes), que la submersion annuelle ne semble pas gêner, mais dont le bois tendre ne résiste guère aux glaces. C'est peutetre pour cette raison que le Thuja occidentalis, - si bien acclimaté aux marécages, réussit rarement à s'implanter sous le haut niveau normal des cours d'eau. Les autres conifères - par exemple le pin blanc (Pinus Strobus) - - tolèrent très mal la submersion. Beaucoup d'arbres croissent uniquement au-dessus des plus hauts niveaux atteints par l'eau.

Les arbres croissant sous le haut niveau normal ont parfois un port caractéristique, ainsi les troncs en forme de bouteille ou sur échasses. Chez le dernier type, une grande partie du système radiculaire dépasse le niveau du sol. Cela pourrait indiquer qu'il y a eu érosion ou bien que la plantule, née sur une butte ou sur une souche en décomposition, s'est fixée à demeure en plongeant dans le sol des racines en arcboutant. Un orme ainsi équipé … et croissant où il n'a pu y avoir d'érosion depuis fort longtemps -.., n'avait pas moins d'une centaine d'années. Il existe aussi un type de souche dont le facies est caractéristique des arbres submergés périodiquement : le tronc noueux, tordu, bossué, se prête plus à la photographie qu'à la description.

Pour déterminer l'âge des arbres poussant sous la ligne du haut niveau, il faut établir pour. les différentes espèces étudiées, dans une région donnée, un taux de croissance moyen des couches annuelles du bois. Dans un secteur, l'étude des arbres révélait, pour dix centimètres de diamètre, l'âge suivant: chêne blanc, 22 à 32 ans; érable rouge, 19 à 26 ans; liard du Canada, 14 à 18 ans; orme blanc, 50 à 55 ans. Ailleurs, l'orme pourrait donner un taux beaucoup moins élevé; celui-ci me paraît nettement exceptionnel.

6. Phanérogames: arbustes. -... Plusieurs arbustes sont des commensaux habituels de la grève; d'autres ne vivent qu'à l'abri de la submersion. Parmi les espèces des berges et des grèves du secteur étudié, il y a lieu de citer : Alnus incana, Cornus Amomum, Cornus stolonifera, Celastrus scandens, divers Crataegus, Menispermum canadense, Myrica Gale, Parthenocissus quinquefolia, Physocarpus opulifolius. Rosa blanda, Rosa johannensis, Rhus radicans, de nombreux saules arbustif's, notamment Salix Bebbiana, S. lucida, $S$. cordata, $S$. longifolia, $S$. lucida, S. petiolaris, S. rigida, Spiraea latifolia, Vitis riparia. 'Tous n'ont pas le même rôle écologique. Certains sont absolument palustres. Un propriétaire de la région de Montréal, ayant prétendu que son terrain était devenu un marécage par suite de la construction d'un canal, il fut possible de démontrer que ces conditions sévissaient antérieurement grâce à un saule palustre, dont il avait été possible de déterminer l'âge au moyen des conches annuelles du bois. D'anciennes cartes datant d'un siècle vinrent d'ailleurs, par la suite, confirmer l'existence à cet endroit d'un marécage permanent.

7. Phanérogames : plantes herbacées. Des centaines d'espèces du Québec sont susceptibles d'être évoquées ici. Le secteur de rivière cité à titre d'exemple ne comprenait pas moins de soixante espèces ripariennes, sans compter un très grand nombre d'espèces proprement aquatiques et de plus nombreuses nettement hostiles à la submersion. Il suffira donc de quelques exemples pour illustrer les diverses catégories.

A. -- Plantes aquatiques ou définitivement palustres. - A titre d'exemples, Eriocaulon septangulare (j'ai découvert toutefois au lac Mistassini une espece nouvelle pour la science, Eriocaulon Rollandii Rousseau, habitant les grèves émergées tout l'été), les Nuphar et $\mathrm{Nym}$ phaea, Pontederia cordata, de nombreux Potamogeton, Myriophyllum et Sparganium, le Vallisneria, Lobelia Dortmanna, Nymphoides lacunosum, Brassenia peltata, etc.

B. - Plantes ripariennes. - Celles-ci entrent dans deux catégories :

a) Des plantes aquatiques tolérant une courte émersion estivale, notamment des espèces de Sagittaria et Polygonum, Scirpus validus et Scirpus fluviatilis, etc. Elles peuvent crô̂tre dans la partie inférieure de la grève découvrant peu de temps au cours de l'été, parce qu'il y sévit alors des conditions palustres permanentes.

b) Les plantes ripariennes proprement dites ou d'habitats normalement marécageux. A titre d'exemples: Agrostis palustris, Apocynum sibiricum, A pios americana, Aster ontarionis, Calamagrostis canadensis, Carex typhina, Carex Oederi ou Carex viridula dans l'estuaire, Che- 
lone glabra, Cyperis dentatus et Cyperus diandrus, Desmodium canadense, Habenaria flava, Heleniam autumnale, Lobelia Kalmii, Lythrum. salicaria, Lysimachia terrestris, Potentilla anserina, Ranunculus flammula, des espèces de Scutellaria, Xanthium, Hypericum et Mentha. En dehors des grèves, toutes ces plantes peuvent habiter des endroits qui sont marécageux une partie de l'année. Certaines, comme le Potentilla anserina, ont des écotypes habitant chacun un habitat différent. La plupart de ces espèces peuvent à l'occasion pousser daus des habitats non ripariens ni palustres, mais elles ne constituent pas alors des formations groupant un grand nombre des éléments précédents.

C. - Plantes indifférentes, pouvant pousser aussi bien dans la zone riparienne, périodiquement inondée, que sur la berge au-dessus el dans d'autres habitats non aquatiques. Ainsi la prèle des champs (Equisetum arvense); mais en réalité, l'espèce comprend de nombreux écotypes particuliers à des habitats différents. La prèle se comporte un peu comme le Potentilla anserina, cité plus haut, mais en ofrrant un plus grand nombre d'écotypes. Dans la catégorie des indifférentes, se placent aussi les Melilotus et Oenothera, dont les grandes colonies sont si caractéristiques de la zone riparienne. Dans l'ensemble, ce sont plus des plantes de pleine lumière que des plantes ripariennes.

D. - Espèces annuelles ou bisannuelles, appartenant à des habitats non aquatiques, mais trouvant sur la grève émergée l'été une saison de végétation assez longue pour leur permettre de pousser. Notons Ambrosia artemisiifolia, Erigeron canadensis, Setaria viridis, Plantago major, etc. Sur l'île Anticosti, une florule arctique-alpine vivace (comprenant notamment: Arctostaphylos rubra, un arbuste rampant) pousse sur les platières des rivières émergées au cours de l'été; ce sont des plantes de pleine lumière, à cycle annuel très court, et dont la structure permet la submersion, surtout avant l'éveil de la végétation.

E. - Enfin, la liste innombrable des plantes hostiles à la submersion, et parmi lesquelles il suffira de citer Sanguinaria canadensis, Monotropa uniflora, les Trillium, Erythronium americanum, etc.

\section{III. - Les facteurs inhibiteurs}

La lutte écologique repose sur la mise en worre de facteurs inhibiteurs on léthaux. Les seuls qu'il y a lieu de mentionner ici dépendent de l'activité humaine et surtout de l'industrialisation.
1. Dans les villes chargées de fumées de charbon et de mazout, les conifères poussent mal et encore plus difficilement les lichens corticaux.

2. Sur la rivière Outaouais, dans le voisinage des pulperies oì l'air est empesté de vapeurs sulfureuses, ces mêmes lichens font complètement défaut ou ne présentent plus de manchons denses.

3. La flore aquatique de l'Outaouais a été considérablement affectée par les égouts de deux grands centres urbains, Ottawa et Hull. Des eaux gluantes inondent la partie supérieure de la grève et perturbent la croissance de quelques éléments ripariens. La forte teneur en matières azotées favorise parfois la croissance épidémique d'algues. Ainsi, dans la région de Montréal, un Melosira (diatomée) se multiplia à un tel point que les filtres des aqueducs nécessitèrent un entretien spécial pendant un certain temps; de mème la croissance surabondante de Cladaphora, pendant près d'un mois, boucha complètement les prises d'eau d'aqueducs. Cette prolifération, dans les deux cas, résultait de l'interaction de l'insolation continue pendant plusieurs jours, d'une température donnée, et de l'abondance d'engrais.

4. Il faudrait aussi tenir compte de certains facteurs physiques, notamment l'érosion causée par le flottage des bois ou des inondations provoquées par des facteurs humains, et qui perturbent la végétation.

\section{IV. - Comparaison des cotes provenant des relevés botaniques et de l'échelle d'étiage \\ (Voir tableau page 668.)}

Pour la compréhension du tableau, il faut tenir compte des points suivants, auxquels réfèrent les chifres et lettres entre parenthèses sur le tableau : (1) les localités des deux rives sont distinguées par les lettres $(x)$ et $(y)$ et sont citées dans un ordre consécutif, d'aval en amont. (2) Cette ligne de niveau, déterminée au moyen de la végétation, correspond dans ce secteur à un débit de $1455 \mathrm{~m}^{3} / \mathrm{s}$ à la station A. Lieau ne descend plus bas que très peu de temps; le sol de cette bande du lit reste alors si imprégné d'eau que la végétation aquatique s'y maintient. - (3) L'emplacement de l'échelle d'étiage et la station où a été poursuivie l'étude botanique coïncident rarement, les conditions écologiques ne s'y prêtant pas (constructions, érosion, srève débarrassée de la végétation naturelle, etc.). L'éloignement de la station de comparaison, par. fois meme sur l'autre rive, explique souvent les 


\section{Tablead I}

Etude comparée des niveaux obtenus à l'échelle d'étiage et des niveaux basés sur la végétation (Pour linterprétation des lettres ct chiffes entre parenthèses, voir le t'exte.)

\begin{tabular}{|c|c|c|c|c|c|c|c|c|c|c|c|c|c|}
\hline $\begin{array}{l}\text { Localités } \\
\text { (1) }\end{array}$ & $\begin{array}{c}\text { Le plus } \\
\text { bas debit } \\
\text { observé } \\
\text { Echelle } \\
\text { d'etiage } \\
651 \mathrm{~m}^{\prime \prime} / \mathrm{s} \\
(6) \\
(3-5 / 3 / 34) \\
(\mathrm{en} \mathrm{m}) \\
\end{array}$ & $\begin{array}{l}\text { Débit de } \\
840 \mathrm{~m}^{3 /} / \mathrm{s} \\
\text { Echelle } \\
\text { d'étiage } \\
\text { (3) (6) (7) } \\
(\mathrm{en} \mathrm{m}) \\
\end{array}$ & $\begin{array}{c}\text { Niveau su- } \\
\text { périeur de } \\
\text { l'habitat } \\
\text { aquatique } \\
\text { permanent, } \\
\text { basé sur } \\
\text { végetation } \\
(2) \\
(\text { en } m) \\
\end{array}$ & $\begin{array}{c}\text { Débit de } \\
1455 \mathrm{~m}^{3 / \mathrm{s}} \\
\text { Echelle } \\
\text { d'étiage } \\
(3) ;(6) \\
(\text { en } \mathrm{m}) \\
\end{array}$ & $\begin{array}{c}\text { Submersion } \\
\text { annuelle } \\
\text { réguliere, } \\
\text { indiquee } \\
\text { par } \\
\text { végétation } \\
\\
\text { (cn m) } \\
\end{array}$ & $\begin{array}{c}\text { Débit de } \\
4145 \mathrm{~m}^{3} / \mathrm{s} \\
\text { Echelle } \\
\text { d'étiage } \\
\text { (3) (6) } \\
\\
\text { (en m) } \\
\end{array}$ & $\begin{array}{c}\text { Haut niveau } \\
\text { normal } \\
\text { ordinatre } \\
\text { basé sur } \\
\text { végétation } \\
\\
\text { (en m) } \\
\end{array}$ & $\begin{array}{c}\text { Débit de } \\
6050 \mathrm{~m}^{3} / \mathrm{s} \\
\text { Echelle } \\
\text { d'étiage } \\
\text { (3) (6) (8) } \\
(\mathrm{en} \mathrm{m}) \\
\end{array}$ & $\begin{array}{c}\text { Débit de } \\
5600 \mathrm{~m}^{2} / \mathrm{s} \\
\text { Echelle } \\
\text { d'etiage } \\
(3)(6)(8) \\
(\mathrm{en} \mathrm{m})\end{array}$ & $\begin{array}{c}\text { Très haut } \\
\text { niveau } \\
\text { basé sur } \\
\text { végétation } \\
\\
(\mathrm{en} \mathrm{m}) \\
\end{array}$ & $\begin{array}{c}\text { Débit de } \\
8735 \mathrm{~m}^{3} / \mathrm{s} \\
\text { Echelle } \\
\text { d'étiage } \\
(3)(6)(9) \\
(\mathrm{en} \mathrm{m}) \\
\end{array}$ & $\begin{array}{c}\text { Débit de } \\
7800 \mathrm{~m}^{3} / \mathrm{s} \\
\text { Echelle } \\
\text { d'etiage }^{\prime} \text { (3) (6) (9) } \\
\\
\text { (en m) } \\
\end{array}$ & $\begin{array}{c}\text { Le plus } \\
\text { haut débit } \\
\text { observé } \\
\text { Echelle } \\
\text { d'étiage } \\
9128 \mathrm{~m}^{3} / \mathrm{s} \\
(6) \\
(17 / 4 / 51) \\
(\text { en m) } \\
\end{array}$ \\
\hline$A(x) \ldots$ & 38,90 & 39,10 & 39,62 & 39,64 & 40,90 & 41,16 & 42,03 & 42,00 & 41,81 & 43,16 & 43,13 & 42,72 & 43,23 \\
\hline $\mathrm{B}(y) \ldots$ & & 39,10 & 39,65 & 39,64 & 40,90 & 41,16 & 42,03 & & 41,81 & $?$ & & 42,72 & \\
\hline $\mathrm{C}(y) \ldots$ & & 39,10 & 39,65 & 39,65 & 40,96 & 41,19 & 42,03 & & 41,85 & 43,13 & & 42,77 & \\
\hline $\mathrm{D}(y) \ldots$ & & 39,11 & 39,71 & 39,65 & 40,96 & 41,24 & 42,06 & & 41,91 & 12,94 & & 42,86 & \\
\hline $\mathrm{E}(x) \ldots$ & & 39,11 & 39,68 & 39,65 & 41,36 & 41,24 & 42,06 & 42,15 & 41,91 & 43,37 & 43,25 & 42,86 & \\
\hline$F(g) \ldots$ & & 39,13 & 39,74 & 39,67 & 41,39 & 41,32 & 42,18 & & 42,01 & 43,04 & 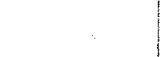 & 43,00 & \\
\hline $\mathrm{G}(x) \ldots$ & & 39,13 & 39,74 & 39,67 & 41,42 & 41,32 & 42,15 & 42,24 & 42,01 & 43,31 & 43,45 & 43,00 & \\
\hline$H(x)(5)$ & & 39,13 & 39,74 & 39,70 & 40,90 & 41,39 & 42,24 & 42,43 & 42,14 & 43,55 & 43,65 & 43,17 & \\
\hline$I(y) \ldots$ & & 39,13 & 39,71 & 39,70 & 41,30 & 41,43 & 42,24 & 42,43 & 42,16 & 43,52 & 43,65 & 43,20 & \\
\hline$J(y) \ldots$ & & 39,16 & 39,77 & 39,80 & 41,42 & 41,59 & 42,27 & & 42,35 & 43,49 & & 43,45 & \\
\hline$K(x) \ldots$ & & 39,17 & 39,80 & 39,82 & 41,51 & 41,60 & 42,33 & & 42,39 & 43,52 & & 43,49 & \\
\hline $\mathrm{L}(x)$ & & 39,21 & 39,89 & 39,92 & 41,54 & 41,74 & 42,88 & 42,88 & 42,56 & $\begin{array}{c}\text { Entre } \\
43,80 \\
\text { et } 43,95\end{array}$ & 44,25 & 48,74 & \\
\hline$M(y) \ldots$ & 38,94 & 39,21 & 39,86 & 39,92 & 41,69 & 41,72 & 42,88 & 42,88 & 42,54 & 43,77 & 44,25 & 43,71 & 44,25 \\
\hline$N(x)$. & & 39,28 & 39,92 & 39,97 & 41,90 & 41,94 & 43,25 & 43,34 & 42,79 & 44,29 & 44,88 & 44,04 & \\
\hline$O(x)$ & 39,24 & 39,47 & 40,29 & 39,97 & 42,18 & 42,46 & 43,71 & 43,68 & 43,43 & 44,56 & 45,33 & 44,82 & 45,39 \\
\hline
\end{tabular}


divergences. - (4) Le très haut niveat n'est pas toujours décelable au moyen des éléments botaniques dans une région où l'air est imprégné des vapeurs sulfureuses des pulperies et les eaux polluées par les égouts des grandes agglomérations urbaines. A cause de cela, le niveau indiqué est parfois inférieur au haut niveau absolu décelé par l'échelle d'étiage. - (5) La station $H$ se trouve au fond d'un lac communiquant avec la rivière par un étroit goulet. L'échelle d'étiage servant de base de comparaison se trouvait à distance, sur l'autre rive. (6) Les débits indiqués sont ceux de la station A. - (7) Comme il y a peu de données sur la courbe d'élévation correspondant au débit de $650 \mathrm{~m}^{3} / \mathrm{s}$ à la station $A$, j'ai indiqué celui de $840 \mathrm{~m}^{3} / \mathrm{s}$, qui représente un bas débit assez normal. - (8) Le débit de $6050 \mathrm{~m}^{3} / \mathrm{s}$ constitue une meilleure base de comparaison que celui de $5600 \mathrm{~m}^{3} / \mathrm{s}$, mais ne pouvant produire dans ce cas toutes les données nécessaires à l'édification de la courbe, celle du débit de $5600 \mathrm{~m}^{3} / \mathrm{s}$ à la station A facilitera la comparaison. - (9) Comme précédemment, le débit de $8735 \mathrm{~m}^{3} / \mathrm{s}$ constituerait une meilleure base de comparaison que celui de $7800 \mathrm{~m}^{3} / \mathrm{s}$, mais ce dernier fournira néanmoins une base de comparaison utile.

Les niveaux tirés des formations végétales et ceux des echelles d'étiage, pour le haut niveau normal ordinaire surtout, coincident remarquablement : la divergence est de 3 à $9 \mathrm{~cm}$ (sauf à la station aberrante $H$ et à la station 1 ). Cette légère difiérence pent être imputable aux facteurs suivants :

1. Absence de concordance des échelles d'étiage et des stations régétales étudiées. Le niveau d'une rivière n'est pas nécessairement le même sur les deux rives. De même, le niveau des élargissements lacustres peut varier beaucoup d'une extrémité à l'autre. Les relevés botaniques m'ont révélé une fois une discordance de $21 \mathrm{~cm}$ sur unc longucur de $15 \mathrm{~km}$, le long d'un élargissement lacustre du Saint-Laurent dans la région de Montréal. L'eau Lès calme aurait pu laisser prévoir un niveau sensiblement le même d'amont en aval. Les données des échelles d'étiage vinrent par la suite confirmer les cotes fournies par la végétation.

2. La végétation ne se réveille pas exactement en mème temps sur tout le parcours, d'environ cent kilomitres.

3. Les vents locaux peuvent élever localement le niveau de l'eau et amplifier à des endroits le mouvement de la vague. - L'ampleur du clapotis de la vague varie avec la structure physique du rivage.

Malgré ces facteurs de discordance, la dévia- tion reste habituellement faible. La méthode de détermination du haut niveau normal, exposée plus haut, constitue donc une technique sûre. Dans tous les cas, très nombreux, où j'ai eu à déterminer le niveau de l'eau, j’ai toujours établi mes coles sans rien savoir des données des échelles d'étiage. C'est seulement une fois le travail terminé que j'établissais la corrélation, qui s'est toujours révélée adéquate. Avec les annécs, la plus grande partie du réseau fluvial de la région de Montréal a été soumise à cette expertise botanique, mais par secteurs discontinus. Une fois toutes les pièces du casse-tête chinois en place, la corrélation entre les éléments s'est montrée parfaite.

\section{E- Effets de l'élévation du haut niveau normal}

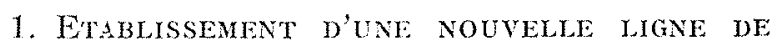
RIVAGe. - II faut des années pour détruire les débris ligneux submergés. Si ces fragments végétaux ne sont pas exposés à l'air, ils restent pratiquement imputrescibles et soumis aux lents processus de la fossilisation. Mème à la surface de l'eau, plus de vingt ans après la construction d'un barrage, des souches témoignent encore de l'existence de l'ancienne forêt. Théoriquement, l'élévation du niveau par une chaussée crée un nouveau rivage, sujet aux mêmes fluctuations qu'avant la construction; mais il n'en est pas ainsi en pratique. Le niveau de l'eau fluctue avec les besoins des entreprises hydrauliques. A l'époque des crues, l'ouverture du barrage déverse le trop-plein. A des baisses subites de quelques jours, peuvent succéder, en plein été, des périodes pendant lesquelles le niveau de l'eau derrière la chaussée atteint le maximum. Sur le plan floristique, chaque cas présente un problème particulier; mais fréquemment, l'étroite grève fluctuante, couverte de débris végétaux en dócomposition, ressemble à un dépotoir s'opposant à l'établissement d'une nouvelle formation riparienne équilibrée.

2. LA NoUVELLE NAPPE PHRÉATIQUE ET LA VÉGÉThTION. -... Lorsque la nappe phréatique, au bord de la rivière, suit le niveau de l'eau, la construction d'une chaussée l'affecte aussitôt. Des puits gui avaient l'habitude de tarir au cours de l'été sont désormais améliorés. Les conditions écologiques même au-dessus du nouveau haut niveau, sont parfois changées au point de modifier l'usage et le rendement des terrains.

A. - Gazons aboisinanl la nouvelle ligne de rivage. - En montant dans le sol, la nappe d'eau favorise le gazon, en maintenant une 
atmosphère humide à des endroits soumis autrefois à la sécheresse. L'eau de capillarité provenant de la nouvelle nappe phréatique suffit parfois aux gazons et élimine les arrosages estivaux, souvent nécessaires jusque-là. La nouvelle situation, par contre, peut être une cause d'ennuis, que l'on comprend mieux en examinant d'abord ce qui se passe au printemps dans le Québec, lors de la ionte des neiges. Par suite de l'hiver rigoureux, le gazon ne reste pas verdoyant pendant l'hiver, contrairement à ce qui se passe en France. Le parterre jaune grisâtre, qui succède à la couche de neige, pourrait laisser croire que le froid y a tué toute végétation; mais l'appareil souterrain est resté vivace : moins de deux jours après la fonte des neiges, la verdure réaparaît brusquement. A ce moment, le gazon est littéralement détrempé. Tant qu’il n'y a pas eu essorage du sol, il ne peut tolérer le piétinement, qui le transformerait en bourbier. Une fois l'excès d'eau disparu et le gazon en pleine vigueur, il n'y a plus de problème; il ne reste plus qu'un terrain superficiellement desscehé, mais renfermant de l'eau de capillarité adhérant aux particules terreuses. Les gazons avoisinant les nappes d'ean surélevées, s’ils sont trop imbibés, peuvent se comparer aux parterres au printemps et ne plus tolérer la promenade.

B. - Champs en bordure de la nouvelle ligne de rivage. - Dans les conditions ordinaires, les propriétaires laissent habituellement une étroite bande de terrain inexploitée entre le champ en culture et la rivière. Elle se réduit habituellement à la berge. Après l'établissement d'une nouvelle ligne de rivage - surtout si le terrain est bas -, il y aurait avantage à ne pas continuer la culture jusqu'à la rivière et à laisser s'établir en bordure du cours d'eau un mince ruban de végétation spontanée. Ce secteur protégerail le terrain contre l'érosion au cours des débordements.

C. - Páturages. - Par le piétinement des sabots, les besliatux el autres herbivores endommagent le tapis de végétation spontanée. Le pâturage par roulement prévient la transformation du champ en bourbier, qui serait fatale autrement. Le broutage détruit les sous-bois. Les sols non égouttés sont particulièrement vulnérables. Dans un cas particulier que j'ai étudié, le pacage avait transformé le sous-bois en marécage. Une chausse maintenait la nappe d'eau au haut niveau normal. Comme elle ne débordait pas de l'ancien lit, il n'y avait pas eu lieu d'exproprier des bandes de terrain des propriétés riveraines. Toutefois, une situation qui n'existait jusque-là qu'au printemps se maintenait toute l'année. II n'y avait plus cette alternance annuelle d'un haut niveau normal et d'un bas niveau normal qui, en facilitant l'égouttement du sous-sol, avait une action bénéfique à cet endroit. I.e lot de villégiature aurait pu ne pas subir les inconvénients de cet exhaussement estival : les citadins reviennent dans leur maison d'été à une époque où la végétation est en pleine activité et où la surface du sol est essorée. Des hommes auraient pu marcher alors sans inconvénients sur le terrain, à $60 \mathrm{~cm}$ au moins au-dessus de la nappe phréatique; mais le pacage inconsidéré au printemps, avant le retour du propriétaire, eut vite fait de défoncer la couche superficielle et d'atteindre la nappe d'eau permanente. La propriété devint un immonde bourbier. Lors de la construction de barrages, il serait dans l'ordre de réviser les conditions de pacage au bord des cours d'eau.

D. - Forèt. - On l'a vu précédemment, des arbres tolèrent une submersion printanière de la souche et même, dans certains cas, poussent spontanément dans des marécages ou sur les grèves périodiquement submergées. D'autres espèces, par contre, sont intolérantes à la submersion et l'élévation du niveau de la nappe phréatique les fait mourir. Les conifères de l'est du Canada sont dans ce cas - si l'on excepte le Thuja occidentalis dont un écotype vit dans les marécages. L'élévation de la nappe d'eau peut donc affecter sérieusement des parcelles forestières, même à distance de la nouvelle ligne de rivage, lorsque les terrains sont bas.

E. - Formation et élimination de marécages. - Un secteur important d'une rivière du Québec coule entre des rivages bas, passant insensiblement à une formation forestière marécageuse. La corniche littorale, également plane et couverte d'une mince couche d'eau, favorise la croissance d'une large batture couverte de plantes aquatiques. La rive n'est guère accessible par terre ou par eau et ne se prête pas aux établissements estivaux. Survienne la construction d'un barrage : l'élévation du niveau de la rivière fera disparaitre des marécages; la vague atteindra désormais une pente plus abrupte, où se formera une nouvelle grève plus favorable aux demeures de villégiature. Les conditions édaphiques favoriseront mème, par endroits, la naissance spontanée de plages sablonneuses. En dautres points, par contre, il pourra se constituer de nouveaux marécages. L'amplitude annuelle de la nouvelle nappe d'eau sera si faible qu'il sera probablement possible de prévoir, moins de deux ans après l'élévation du niveau, l'orientation définitive des communautés végétales.

L'élévation de la nappe phréatique crée donc des conditions écologiques qui, selon les cas, favoriseront ou défavoriseront les propriétaires riverains. 